\title{
HACIA LA COMPRENSIÓN DEL FENÓMENO DE LA SORDEDAD: HABITUS COMO PROPUESTA EPISTEMOLÓGICA
}

\author{
TOWARDS UNDERSTANDING THE PHENOMENON OF \\ DEAFHOOD: HABITUS AS AN EPISTEMOLOGICAL PROPOSAL
}

\section{KARINA MUÑOZ VILUGRÓN*, ALEJANDRA SÁNCHEZ BRAVO ${ }^{* *}$}

\section{RESUMEN}

En el ámbito de la sordera el predominio del enfoque médico ha generado prácticas profesionales contradictorias y eventualmente poco útiles, que tienden a restringir el desarrollo de la lengua y la cultura de la comunidad sorda limitando la plena participación ciudadana. Considerando el reconocimiento mundial de la cultura e identidad sorda, este trabajo propone avanzar en la comprensión de esta problemática a través del estudio del concepto de Sordedad y su potencial base epistemológica el Habitus. Progresar en este camino puede facilitar la reflexión profesional y las ideas que sustentan sus prácticas y, con esto, la inclusión social efectiva de las personas sordas.

Palabras clave: Sordedad, habitus, comunidad sorda, enfoque socio-antropológico.

\section{ABSTRACT}

In the field of deafness, the predominance of the medical approach has generated contradictory and possibly unhelpful professional practices, which tend to restrict the development of the language and culture of the deaf community and limit full citizen participation. Considering the global recognition of deaf culture and identity, this paper proposes to advance the understanding of this problem by studying the concept of

* Doctora (c) en Ciencias de la Educación, Universidad Austral de Chile. Puerto Montt, Chile. Correo: karina.munoz@uach.cl

** Doctora en Diseño, Orientación e Intervención Psicopedagógica, Universidad Austral de Chile. Puerto Montt, Chile. Correo: asanchezb@spm.uach.cl 
Deafhood and its potential epistemological basis called Habitus. Following this path can facilitate reflection and ideas that support professional practices, and with this, the effective social inclusion of deaf people.

Keywords: Deafhood, habitus, deaf community, socio-anthropological approach.

Recibido: 27.02.17. Aceptado: 24.07.17.

\section{INTRODUCCIÓN}

$\mathrm{E}$ N ChILE Y EN EL MUNDO, la fuerte dicotomía entre el paradigma médico y el socio-antropológico para abordar y comprender la sordera y sus particularidades ha nutrido la investigación científica a partir de sólidos argumentos y resultados, existiendo actualmente estos dos marcos interpretativos dominantes. Esto ha generado como consecuencia la polarización entre profesores, médicos, asistentes sociales y terapeutas quienes, desde distintos ámbitos, ejercen su labor profesional junto a la comunidad sorda.

En términos generales, se sostiene que el modelo médico asume la sordera como una discapacidad e incorpora la intervención asistencialista, terapéutica y rehabilitadora para sanar la enfermedad de la sordera, es decir, la persona es considerada "no oyente" (Moeller, White \& Shisler 2006). Por otro lado, el paradigma socio-antropológico sostiene que los sordos pertenecen a una cultura minoritaria o minoría étnica (Lane, 2005) que posee una lengua propia (lengua de señas) y que sus miembros desarrollan una identidad particular; por lo tanto, son considerados personas sordas (descartando persona no oyente), destacando a un sujeto con derechos que está inmerso en una sociedad que naturalmente no es intercultural. En este aspecto Becerra-Sepúlveda (2013) propone transformar las relaciones sordos-oyentes en oportunidades de intercambios sociales que permitan a la persona sorda superar una histórica posición de subalternidad, caracterizada por la invisibilización de su cultura e historia y la persistencia de la cultura oyente por imponer, desde su perspectiva, soluciones al "problema" de la sordera, soluciones que, según Cuevas, sostienen y perpetúan el colonialismo oyente que genera "un efecto a la vez moralizante, estructurante y opresivo en la formación del sordo" (2013, p. 703).

Cifras mundiales señalan que el 5\% de los niños sordos son hijos de padres sordos, el $95 \%$ restante nacen en familias de padres oyentes con poca o ninguna experiencia previa acerca de la sordera (Lederberg, Schick \& Spen- 
cer, 2013), por lo tanto, requieren diversos tipos de apoyos para atender las necesidades específicas del nuevo miembro de la familia. En este sentido, ambos paradigmas acogen las recomendaciones referidas a la detección precoz, la intervención temprana, el seguimiento continuo del desarrollo comunicativo, lingüístico, motor, cognitivo y socioemocional del niño sordo; también, la protección de los derechos del niño y su familia a través de la elección informada, la toma de decisiones y el consentimiento (Department of Health and Human Services, 2009; Early Hearing Detection and Intervention Information \& Resource Center, 2004; Joint Committee on Infant Hearing, 2007; Organización Mundial de la Salud, 2015). No obstante, el problema se suscita cuando los padres de niños sordos comienzan a ser asesorados por profesionales que difieren en formación y actitudes, poseen más o menos niveles de conocimiento sobre el tema e interpretan la sordera desde uno u otro paradigmas o por una combinación de estos, asesoramiento que más que ayudar a los padres les puede generar incertidumbre, falsas expectativas o el ejercicio de prácticas contradictorias o escasamente provechosas. Un ejemplo de esto es cuando desde el ámbito médico se sugiere implante coclear y desde el ámbito educativo se indica escolarización para el desarrollo de la cultura e identidad sorda. El primero lleva implícita la recuperación de la audición y con ello el desarrollo del lenguaje oral, de carácter auditivo-verbal, mientras que la segunda indicación implica la inmersión en una cultura minoritaria donde uno de los principales aspectos diferenciadores es el aprendizaje de una lengua viso-gestual. Las características visuales de la lengua de señas permiten el desarrollo de la visualidad. En el discurso en lengua de señas la mirada sostenida (eye gaze) se utiliza para regular la toma de turnos, los cambios de rol y tiene un carácter sintáctico en la lengua de señas (Metzger, 1998). Si bien este enfoque excluye la formación auditivo-oral, sí manifiesta apertura al uso de tecnologías, las cuales corresponden a una decisión personal del individuo sordo.

Otro ejemplo que ilustra la situación que viven los padres de niños sordos se presenta cuando ambos paradigmas sostienen que los niños sordos deben desarrollar la lengua en los primeros años de vida y así evitar un retraso en el desarrollo del lenguaje que podría transformarse en una situación definitiva. Desde la postura médica/rehabilitadora se sugiere a los padres dedicar grandes esfuerzos a la adquisición del habla de su hijo sordo mediante terapia auditivo/verbal y se les recomienda que la lengua de señas sea elegida solo como último recurso (Johnston, 2006; Petitto, 1998), ya que la mejor forma de adquirir la lengua oral sería sin la lengua de señas. 
Desde el paradigma socio-antropológico, se indica que los niños sordos deben aprender la lengua de señas en los primeros años, debido a que con una primera lengua de base (la lengua de señas) el niño puede llegar a ser bilingüe (en la forma escrita de la lengua oral de su entorno y, tal vez, en la forma hablada), acumulando así los beneficios del bilingüismo (Humphries et al., 2012).

Ante tal problemática, aun cuando estos dos paradigmas mantengan el statu quo que los separa, quizás una forma adecuada de avanzar en la comprensión y forma de abordar la sordera y sus particularidades sea que los profesionales involucrados reconozcan la dificultad que han tenido para resolver los propios conflictos teóricos y prácticos que están obstaculizando el desarrollo de soluciones pertinentes a problemas reales y urgentes de la población sorda. Por ejemplo, temas perentorios y de gran relevancia: cómo incrementar el nivel lector en las personas sordas, cómo estimular el desarrollo del pensamiento y la resolución de problemas, son temas que han sido estudiados; sin embargo, el conflicto se basa en la modalidad de lengua utilizada y en los instrumentos aplicados para medir tales capacidades. Otros temas que emergen son: cómo proveer el acceso a quien requiera y decida el uso de implantes cocleares; cómo legitimar las lenguas de señas; cómo desarrollar una política de salud orientada a la detección temprana de hipoacusia y el acceso a adecuados procesos de re-habilitación; cómo conciliamos el respeto por la comunidad de personas sordas, su lengua, su cultura (Padden \& Humphries, 1998, 2005), sin desmerecer los avances tecnológicos que pueden mejorar la calidad de vida de estos.

En el contexto chileno es imprescindible considerar que el Estado de Chile reconoce la existencia de la Comunidad de Personas Sordas y la legitimidad de la LSCh (Ley No 20.422/2010), por lo tanto releva la importancia del paradigma socio-antropológico y con ello invita a reflexionar acerca de lo que tenemos como creencias referidas a las personas sordas y sobre las que apoyamos nuestras decisiones.

En consecuencia, el propósito de este trabajo es apoyar esta reflexión mediante el estudio del concepto de Sordedad y su potencial base epistemológica, el habitus. Confiamos que la comprensión de estos constructos podrá contribuir a desarrollar profesionales más sensibles, comprensivos y comprometidos que favorezcan la inclusión de las personas sordas en las distintas esferas de la sociedad. 


\section{SORDEDAD}

Ladd (2003) propone que el conocimiento de la persona sorda no se obtiene estudiando aspectos de la sordera, sino por el conocimiento de su Sordedad. Es así como él propone el término del inglés Deafhood (Sordedad), dejando de lado deafness (sordera).

La sordera la define como una condición médica. Desde el punto de vista terminológico se refiere a "oídos rotos" en necesidad de una cura, es decir, la persona es vista como anormal, mientras que la Sordedad la refiere a diferentes experiencias de la persona sorda en su trayectoria de vida en un contexto oyente. La Sordedad, como construcción teórica, contempla las etapas que cada persona sorda vive en la búsqueda de reencontrarse con su ser, reconocerse como persona sorda, es decir, como alguien distinto al oyente. Para Humphries (2008), la Sordedad es lo opuesto a lo que llama audismo ${ }^{1}$. Así, la Sordedad reconoce que todas las personas sordas se embarcan en un viaje hacia la profundización y el perfeccionamiento de su ser sordo.

Para Ladd (2003) es por medio de la experiencia individual y colectiva donde la persona sorda adquiere su Sordedad, transformándose en su identidad Sorda Individual, la cual es desbaratada por una forma particularmente intensa de opresión educacional.

Las vivencias adquiridas por el sordo junto a su colectivo sordo, más aquellas adquiridas junto a su familia, compañeros y profesores oyentes, con los cuales desarrolla relaciones intersubjetivas impacta en la configuración de su identidad personal dando origen a conceptos y conocimientos que le son propios.

Desde una perspectiva histórica, el proceso de configuración de las sordedades es considerado un proyecto de vida que se inicia con el nacimiento y culmina con la muerte de la persona; en sus distintas etapas este fenómeno recoge los logros pasados y la proyección de la vida futura.

En síntesis, es posible destacar que las percepciones y creencias de cada persona sorda representan sus experiencias de vida, su capital cultural y contribuyen al desarrollo de una identidad particular.

\footnotetext{
${ }^{1}$ Audismo: Es el supuesto ideológico de la supremacía del oyente o de aquellas personas sordas que actúan como oyentes, por sobre los sordos.
} 
El concepto de habitus es una de las contribuciones fundamentales que realiza Pierre Bourdieu a la sociología y a la construcción teórica que propone (Teoría de la acción). Habitus corresponde a la traducción latina que Aquino $^{2}$ y Boecio ${ }^{3}$ dan al concepto aristotélico de hexis ${ }^{4}$ (corporal).

Para Bourdieu (1989, p. 18) el habitus está constituido por disposiciones cognitivas, esquemas (schémes) de percepción, pensamiento y acciones corporales que emergen como "producto de la internalización de las estructuras del mundo social". Por lo tanto, el habitus guía la forma en que las personas perciben, comprenden, aprecian y evalúan el mundo social, incluso el sentir y actuar de una manera determinada (Wacquant, 2005).

La palabra "disposición" parece especialmente conveniente para expresar lo que abarca el concepto de habitus (como sistema de disposiciones): en efecto, expresa de entrada el resultado de una acción organizadora que presenta entonces un sentido muy vecino a palabras como estructura; designa por otra parte una manera de ser, un estado habitual (en particular del cuerpo) y, en particular, una predisposición, una tendencia, una propensión, o una inclinación (Bourdieu, 2001[1973], p. 393).

Aparentemente, el habitus podría ser considerado como algo innato, aunque es sabido que se forma de esquemas de percepción y valoración de una estructura social, que pueden actualizarse como "estructuras estructurantes" (Bourdieu, 1991).

En el sintagma "estructuras estructurantes", estructurantes (verbo) se refiere a que el sistema de disposiciones produce prácticas que hacen que lo social emerja y que tenga sentido para el sujeto. De este modo, las estructuras implican la interiorización de los procesos y elementos sociales, en tanto las estructurantes involucran la generación y estructuración de prácticas en la cultura y en los grupos sociales.

${ }^{2}$ Tomás de Aquino (28 de enero de 1225. 7 de marzo de 1274): teólogo y filósofo, escribe acerca de la metafísica. Acepta y comenta las obras de Aristóteles. Reinterpretó la temática de Aristóteles de habitus como acción o movimiento.

${ }^{3}$ Boecio (480 d. C, s/f Pavía, Italia). Filósofo romano. Quien al igual que Tomás de Aquino reinterpreta el habitus aristotélico de una acción o movimiento, el acto del hacedor.

${ }^{4}$ Hexis se refiere a la manera de actuar y comportarse con una apariencia natural. Hexis corporal se vuelve una disposición permanente, una manera duradera de pararse, de hablar, de caminar, y por ello de sentir y de pensar. 
El habitus no es permanente, va cambiando de acuerdo a las situaciones o períodos históricos (Navarro, 2006). Las posibilidades que el habitus da a través de las vivencias que se van guardando permiten acomodar estas experiencias y, al mismo tiempo, modificar los esquemas con nuevos significados cambiando el habitus, el cual direcciona al sujeto hacia la comprensión del otro.

Estos esquemas generativos, que se encuentran socialmente estructurados, han sido conformados a lo largo de la historia de cada sujeto y suponen la interiorización de la estructura social, del campo concreto de relaciones sociales donde el agente social se ha conformado como tal.

Así, el habitus o las normas socializadas o tendencias que guían el comportamiento y el pensamiento, se crea a través de un proceso social, nunca individual, que conduce a patrones que son duraderos y transferibles de un contexto a otro, se desplaza en relación a los contextos y tiempos específicos. Es decir, el habitus es producto de todos estos condicionamientos que aparecen de la experiencia subjetiva de las personas con otros y el mundo (Bourdieu, 1991).

Por lo tanto, al comprender el habitus como una estructura, se hace visible en las relaciones sociales de los sujetos, funciona como principio generador de prácticas y de representaciones que pueden ser objetivamente adaptadas a su meta, sin suponer el propósito consciente de ciertos fines ni el dominio expreso de las operaciones necesarias para alcanzarlos.

Según Bourdieu (2007 [1987]) el habitus origina prácticas individuales y colectivas, y por ende historia; es el habitus el que asegura la presencia activa de las experiencias pasadas que, registradas en cada organismo bajo la forma de esquemas de percepción, de pensamientos y de acción, tienden, con más seguridad que todas las reglas formales y todas las normas explícitas a garantizar la conformidad de las prácticas y su constancia a través del tiempo. La configuración biográfica, por tanto, alude a que cada individuo se sitúe de una manera particular en el mundo, pues toda su experiencia es única.

Es importante recordar que si bien el sujeto, como señala Bourdieu (1991), reconoce el mundo como evidente, sin pasar por una relación objetiva con él, esta evidencia del mundo tiene como condición de posibilidad el sistema de disposiciones (el habitus), generador de prácticas entre las cuales encontramos las cognoscitivas, articuladas a ese mundo de relaciones intersubjetivas de tal forma de volverlas evidentes. 


\section{HABITUS-SORDEDAD}

Habiendo intentado una aproximación conceptual a los constructos en estudio, en este punto se propone su articulación mediante la exposición de cuatro ideas centrales.

En primer lugar, el habitus supone la existencia de un agente oprimido en esquemas de percepciones, de acciones heredadas pero con la posibilidad de generar variedad y ser creativo. Este conocimiento tiene relevancia cuando es incorporado en los sustentos epistemológicos de la sordera a través del concepto de Sordedad, donde se asume que cada sujeto vive experiencias significativas y asume que otros también las viven, se relaciona con otros pues es posible ponerse en el lugar de otros y aprender de ellos. Esta situación es posible si y solo si el sordo conoce al otro (sordo) en su cultura, en su lengua, permitiéndole derribar prejuicios y reconocer situaciones comunes en el aquí y en el ahora.

La segunda idea, pretende destacar que desde la temprana infancia del sordo, la crianza, la educación recibida, los intereses, deseos y motivaciones de las personas que configuran su entorno, constituyen elementos que aportan a la formación de su personalidad única. Es decir, la situación biográfica del sordo constituye el habitus descrito por Pierre Bourdieu y, en consecuencia, configura el fenómeno de la Sordedad que "funciona en cada momento como una matriz de percepciones, de apreciaciones y de acciones" (Capdevielle, 2011, p. 34). Esta información permitiría construir el proceso de Sordedad de una persona sorda integrando las relaciones intersubjetivas individuales y colectivas que ha experimentado. En este punto es importante señalar que la fenomenología podría ser la metodología de estudio de la Sordedad, a través de la observación y el registro de las vivencias personales y colectivas, permitiendo conocer la realidad en el antes y ahora de las vivencias del sordo.

En tercer lugar, hay que considerar que la persona sorda, a lo largo de su existencia, define esquemas que le son propios que difieren de las personas oyentes, y que se encuentran en las vivencias de su propia Sordedad. Es así como la persona sorda logra intervenir en espacios de la sociedad oyente, pues posee el significado de concepto de mundo basado en los conceptos de "cada uno" y del "otro", aportando activamente a la disminución del audismo.

En atención a lo mencionado, y de acuerdo a los postulados de Bourdieu, la cuarta idea sostiene que se puede percibir la Sordedad como posibilidad de la persona sorda para ir modificándose de acuerdo a nuevas vivencias 
culturales. Muchas formas sociales y culturales sordas pueden asemejarse a las de la cultura mayoritaria; sin embargo, sus expresiones son diferentes, a través de formas que todavía no se han examinado. De acuerdo a lo planteado, las personas sordas se encuentran sumergidas en un campo mayor, en una comunidad oyente que le ha impuesto una forma de vida. Por esta razón, Ladd (2003) señala que las culturas sordas no son sub-culturas en la sociedad mayoritaria, sino culturas por derecho propio pues han creado un conjunto de significados culturales por sí mismos. De acuerdo al habitus de Bourdieu (2007 [1987]) no conformarían sub-culturas puesto que son comunidades que poseen principios generadores de prácticas culturales $y$, como agentes sociales, el colectivo Sordo es un agente social que interioriza esquemas y disposiciones para la acción a lo largo de su vida. Bourdieu hace hincapié en que los distintos habitus son resultado de las distintas posiciones que los sujetos ocupan en el espacio social.

\section{REFLEXIONES FINALES}

El respeto y valoración de la diversidad, la igualdad de oportunidad y la equidad social, valores que han sido defendidos en la sociedad actual, han generado cambios significativos en la atención de la persona sorda; estos procesos de cambio invitan a transitar desde una práctica sustentada en la rehabilitación médica a una que reconoce, respeta y valora la cultura e identidad sorda.

Comprender el fenómeno de la Sordedad nos muestra una nueva forma de abordar el estudio y comprensión de la sordera y, de esta forma, como oyentes, ver la existencia de las personas Sordas como una afirmación de la diversidad de la vida humana, la pluralidad que se puede encontrar no en alguna tierra exótica, sino que justo donde estamos situados (Ladd, 2003).

Las vivencias, percepciones y creencias que desarrolla la persona sorda (el habitus) permiten que desarrolle su cultura e identidad propia. Conocer la Sordedad a través de la idea de habitus planteada por Pierre Bourdieu permite relevar a la persona Sorda destacando su situación particular, que le permite situarse en su experiencia y forjar una identidad y así aportar y enriquecer a la comunidad Sorda; por ejemplo, diseñar prácticas pedagógicas sustentadas en las características culturales de esta comunidad, que ayudarían, a su vez, a potenciar sus capacidades y así lograr mejores resultados académicos (Holcomb, 2010). En este sentido potenciar las capaci- 
dades de los estudiantes sordos a través del conocimiento de su Sordedad se propone como la base de una nueva educación para las personas sordas, pues se basa en la perspectiva de la persona sorda y no en la concepción del oyente. Esta es la situación, a través de su cultura, que la comunidad sorda históricamente ha reclamado, que implica la superación del estatus de subalternidad. Por lo tanto, la perspectiva desde la persona sorda es la que debería guiar el quehacer profesional oyente, posibilitando de esta forma acciones positivas hacia esta población, que se traducirán en una efectiva inclusión social, proponiendo espacios de comunicación donde se reconozcan las diferencias como elementos que enriquecen a ambas culturas, es decir, que los intercambios oyentes-sordos deban comprenderse más bien como encuentros interculturales, otorgando el espacio a la comunidad Sorda en la creación de soluciones a problemas por ellos detectados, en las que son ellos los principales gestores al bridar las vías para superarlos.

Para finalizar, es importante mencionar que los planteamientos desarrollados en este trabajo pueden contribuir a una propuesta educativa que surja verdaderamente desde la episteme del conocimiento Sordo diferenciándose de la propuesta intercultural-bilingüe, que busca incorporar al Sordo a un sistema educativo establecido desde la dominancia oyente, ajustándolo y/o adecuándolo a las necesidades de su cultura, con el propósito de generar una relación "más justa" entre colonizador y colonizado (De la Paz, Marín \& Salamanca, 2006), lo cual no resulta extraño ya que el modelo intercultural-bilingüe surge como respuesta a la opresión de los pueblos originarios y no desde la cultura Sorda. Una propuesta desde la episteme del conocimiento Sordo implica que el espacio educativo reconozca la existencia de la diversidad cultural, que valore y promueva el desarrollo de cada una de ellas, sin tener que compensar o ajustar su crecimiento a una estructura determinada. Sería pertinente que el espacio educativo tuviera tantas visiones y misiones como grupos culturales acoja, así como tantas políticas y prácticas quiera la interculturalidad existente.

Avanzando en aspectos más bien prácticos, por ejemplo: la incorporación de modelos culturales y lingüísticos Sordos debería estar sujeta a la decisión de la comunidad Sorda y no a la decisión oyente (director de Escuela o jefe del Departamento de Educación Municipal); las decisiones respecto de lo educativo también deberían depender de la comunidad Sorda e independientemente de su formación académica, pues se debe aceptar que la experiencia Sorda lo faculta para la toma de decisiones. 


\section{REFERENCIAS}

Becerra-Sepúlveda, C.A.d.L (2013). "Interculturalidad y ciencias de la educación en lengua de señas chilena”. Boletín Científico Sapiens Research, 3(2), 2-7.

Bourdieu, P. (1989). Espacio social y el poder simbólico. Buenos Aires: Gedisa. (1991). El sentido práctico. Madrid: Taurus.

. (2001 [1973]). Oficio del científico. Barcelona: Anagrama.

(2007 [1987]). "Espacio social y poder simbólico", en: Bourdieu, P., Cosas dichas (pp. 127-142). Barcelona: Editorial Gedisa.

Capdevielle, J. (2011). "El concepto de Habitus: con Bourdieu y contra Bourdieu”. Anduli Revista Andaluza de Ciencias Sociales, 10, 31-45.

Cuevas, H. (2013). "El gobierno de los sordos: el dispositivo educacional”. Revista de Ciencia Política, 33(3), 693-713.

De la Paz, M. V., Marín, J. y Salamanca, M. (2006). Educación Intercultural Bilingüe, una propuesta educativa para nuestros alumnos sordos. Recuperado de http://www.culturasorda.eu.

Department of Health and Human Services, Centers for Disease Control and Prevention. "Organizational recommendations and screening guidelines 2009” (2015). Oct. http://www.cdc .gov/NCBDDD/ehdi/ddscreen.htm.

Early Hearing detection and Intervention Information and Resource 2004 (2015). "National Center for Hearing Assessment and Management". $<$ http://www.infanthearing.org/survey/2004statesurvey/index.html >.

Holcomb, T. K. (2010). "Deaf Epistemology: The Deaf way". American Annals of the Deaf, 154(5), 471-477.

Humphries, T. (2008). "Talking culture and culture talking”. Open your eyes: Deaf studies talking. Minneapolis: University of Minnesota Press, 35-41.

Humphries, T.; Kushalnagar, P.; Mathur, G.; Napoli, D.; Padden, C.; Rathmann, Ch. \& Smith, S. (2012). "Language acquisition for deaf children: Reducing the harms of zero tolerance to the use of alternative approaches". Harm Reduction Journal, 9, 1-9.

Johnston, T. (2006). "Response to Comments". Sign Language Studies, 6(2), 225-243.

Joint Committee on Infant Hearing. Principles and guidelines for early hearing detection and intervention programs. Pediatrics 120.898-921. 2007. (2015) 04 de nov. Recuperado de <4hyperwww.pediatrics.org/cgi/doi/10.1542/ peds.2007 2333doi:10.1542/peds.200733>

Ladd, P. (2003). Understanding deaf Culture. In Search of Deafhood. Clevedon, UK: Multilingual Matters.

Lane, H. (2005). "Ethnicity, ethics, and Deaf-World". Journal of Deaf Studies and Deaf Education, 10(3), 291-310.

Lederberg, A.; Schick, B. \& Spencer, P. (2013). "Language and literacy development of deaf and hard-of-hearing children: successes and challenges". Developmental Psychology, 49(1), 15-30. 
Metzger, M. (1998). "Eye gaze and pronominal reference in American Sign Language". In Lucas, C. (Ed.), Pinky Extension \& Eye Gaze: Language Use in Deaf Communities (pp. 170-182). Washington, DC: Gallaudet University Press.

Moeller, M., White, K., \& Shisler, L. (2006). "Primary care phisicians' knowledge, attitudes, and practices related to newborn hearing screening". Pediatrics, 118, 1357-70.

Navarro, Z. (2006). "In Search of Cultural Interpretation of Power". IDS Bulletin, 37(6), 11-22.

Organización Mundial de la Salud (OMS) (2015). Sordera y pérdida de audición. 2015. 04 de nov. 2015. Recuperado de <http://www.who.int/mediacentre/factsheets/fs300/es/>.

Padden, C. \& Humphries, T. (1998). Deaf in America: Voices from a Culture. Cambridge. MA: Harvard University Press.

Padden, C. \& Humphries, T. (2005). Inside deaf culture. Cambridge, MA: Harvard University Press.

Petitto, L. A. (1998). "On the biological, environmental and neurogenetic factors determining early language acquisition: Evidence from signed and spoken languages". Bulletin D'Audiophonologie, XIV(1), 337-348.

Wacquant, L. (2005). "Habitus". Beckert, Jens y Zafirovski (pp. 315-319). Milan: International Encyclopedia of Economic Sociology. 